\title{
Superscan: Superiority of xSPECT/CT over OSEM SPECT/CT in bone scans of prostate cancer patients.
}

\author{
Ew-Jun Chen ${ }^{\mathrm{a}, \mathrm{b}}$, Teik Hin Tan ${ }^{\mathrm{a}, \mathrm{b}}$, Ming Tsuey Chew ${ }^{\mathrm{b}, *}$ \\ ${ }^{a}$ Nuclear Medicine Centre, Sunway Medical Centre, No. 5 Jalan Lagoon Selatan, Bandar Sunway, 47500, Petaling Jaya, Selangor, Malaysia \\ ${ }^{\mathrm{b}}$ Centre for Biomedical Physics, School of Healthcare and Medical Sciences, Sunway University, No. 5 Jalan Universiti, Bandar Sunway, 47500, Petaling Jaya, Selangor, \\ Malaysia
}

\section{A R T I C L E I N F O}

\section{Keywords:}

Prostate cancer

Skeletal

Scintigraphy

Metastasis

XSPECT/CT

SPECT/CT

\begin{abstract}
A B S T R A C T
Prostate cancer is one of the most common cancers affecting men. Bone scan is part of the staging modality commonly used to evaluate bone metastasis. A bone scan with diffused increased skeletal tracer uptake relative to soft tissue, combined with faint renal activity is known as a superscan. However, a primary concern are false negatives associated with bone scans, where diffuse metastasis is indistinguishable on superscans. In this study, we performed xSPECT/CT Bone and standard OSEM SPECT/CT reconstruction algorithm in ten prostate cancer patients with high PSA levels, where they initially seem relatively unremarkable on planar images. All patients with extensive bone metastases showed either relatively unremarkable scans or did not demonstrate the true extent of metastatic burden as seen on planar images. Uptake was further confirmed by the correlative diffuse bone lesions on CT images. Our reports also indicated that XSPECT/CT reconstructed images were far superior in delineating focal areas of osteoblastic bone metastasis, when compared with whole body planar images or SPECT/CT images. The extent of metastatic evidence is delineated with excellent clarification by xSPECT/CT images. We propose that whole body XSPECT/CT image reconstruction, or at least SPECT/CT, should be performed in patients with high PSA levels, along with planar imaging, to improve diagnostic accuracy of bone scans in prostate cancer staging.
\end{abstract}

\section{Introduction}

Prostate cancer is one of the most common cancers affecting men worldwide in 2019 (Siegel et al., 2019). Post-mortem autopsies have demonstrated that skeletal system tends to be the most frequent site for metastases (approximately 80\%) (Imbriaco et al., 1998; Shen et al., 2014). Widespread skeletal metastasis is synonymous with high morbidity and poor prognosis; often accompanied with severe complications such as impaired mobility, pathological fractures, spinal cord compressions and generalised skeletal pain (Keller and Brown, 2004). The metastatic extent of prostate cancer with bone involvement correlates with poorer prognosis and reduced survival rate (Soloway et al., 1988; Sabbatini et al., 1999; Halabi et al., 2003). For early diagnosis of prostate cancer, routine clinical and imaging tools includes diagnostic tests such as serum Prostate-Specific Antigen (PSA) levels, transrectal ultrasound (TRUS), computed tomography (CT) and magnetic resonance imaging (MRI); whereby the invasive nature of TRUS-guided core needle biopsy is the gold standard (De Visschere et al., 2010). Therefore, a non-invasive method for early detection and accurate staging is vital to stop progression to widespread skeletal metastatic disease.

Skeletal scintigraphy, also known as radionuclide bone scan, is a common nuclear medicine imaging modality used to detect skeletal metastases in prostate cancer patients (Pollen et al., 1984; Knudson et al., 1991; Gnanasegaran et al., 2009; Tabotta et al., 2019). With the ability to examine the entire skeleton, bone scan is one of the most frequently performed radionuclide imaging examinations due to its high sensitivity (Love et al., 2003; Tabotta et al., 2019). Therefore, it has better diagnostic value compared to standard-of-care imaging, such as plain radiographs or CT, in detecting early or asymptomatic bone metastasis (Love et al., 2003; Shen et al., 2014; Manohar et al., 2017). This procedure requires a "bone-seeking" agent that allows us to visualise new bone formation or any unusual rate of bone turnover by binding to osteoblasts (Agrawal et al., 2015; Imbriaco et al., 1998), using Technetium99m-methyldiphosphonate (Tc99m-MDP). Images are typically acquired at approximately $3 \mathrm{~h}$ after intravenous injection. This delay between time-of-injection and image acquisition allows for excessive radioisotope clearance from soft tissues and renal system,

\footnotetext{
* Corresponding author.

E-mail address: mtchew@sunway.edu.my (M.T. Chew).
} 
Table 1

Examination and reconstruction protocol.

\begin{tabular}{ll}
\hline Scanner & Siemens Symbia Intevo 16 \\
\hline Tc99m-MDP injected dose & $740 \mathrm{MBq}$ \\
Scan delay & 3 h post-injection \\
Scan parameters & 2D Planar: $12 \mathrm{~cm} / \mathrm{min} ; 256 \times 1024$ matrix \\
& 3D SPECT: 60 views @ 7 secs/view; $256 \times 256$ \\
& matrix \\
& CT: $110 \mathrm{kV} ; 75$ ref. mAs; $5 \mathrm{~mm}$ slice thickness \\
Reconstruction algorithm & OSEM SPECT/CT: 8 iterations; 4 subsets; $12 \mathrm{~mm}$ \\
$\quad$ parameters & Gaussian filter xSPECT/CT Bone: 20 iterations; 1 \\
& subset; $20 \mathrm{~mm}$ Gaussian filter \\
\hline
\end{tabular}

resulting in improved skeletal system visual clarity (Love et al., 2003). Gamma cameras are typically used to obtain planar images for bone scans, but the modern hybrid technology of Single-Photon Emission Computed Tomography (SPECT)/CT allows for faster image acquisition with higher accuracy. In today's modern healthcare system, bone scans are relatively quick, inexpensive and widely available.

A "superscan" is defined as bone scan planar imaging with diffuse increased skeletal tracer uptake relative to soft tissue, combined with diminished or non-existent renal activity (Brenner et al., 2012; Love et al., 2003). It has added diagnostic ability to detect early bone lesion changes anywhere with high sensitivity (more than 80\%) (Manohar et al., 2017). However, a major drawback of superscans are the false negatives associated with bone scans demonstrating diffuse metastases, whereby these scintigraphs are almost indistinguishable on planar images (Agrawal et al., 2015; Al-Tamimi et al., 2012). In addition, superscans may produce inconclusive results and false interpretations due to metabolic bone diseases (Brenner et al., 2012).

This study aims to evaluate the accuracy of a superscan as defined above, with combination of 3D SPECT/CT reconstructed images for detecting and diagnosing bone metastases in prostate cancer patients. Additionally, this study also looks into optimising and enhancing the current skeletal scintigraphy protocols for patients at Sunway Medical Centre, Malaysia.

\section{Methodology}

Ten prostate cancer patients presented with markedly elevated serum PSA levels were included in this study. All patients first underwent routine planar imaging, which was acquired $3 \mathrm{~h}$ after an injection of Tc99m-MDP, with a dose of $740 \mathrm{MBq}$. Subsequently, a hybrid SPECT/CT imaging was performed, consisting of whole-body (vertexof-skull to pelvis) 3D SPECT study, immediately followed by a low-dose CT scan.
All SPECT/CT hybrid images were reconstructed using iterative xSPECT Bone algorithm (developed by Siemens Healthcare) and standard Ordered-Subset Expectation-Maximisation (OSEM) SPECT reconstruction algorithm for visual clarity comparison. Both standard OSEM SPECT and Siemens' xSPECT Bone reconstructions were individually fused with CT images for anatomical localisation and attenuation correction. Examination protocols and reconstruction algorithm parameters are listed below in Table 1. Four of the patients discussed below were representative of the ten cases.

\section{Results}

All patients with extensive bone metastases showed either relatively unremarkable tracer uptake or did not demonstrate the true extent of metastatic burden as seen on planar images (Fig. 1). As shown in Fig. 1, patients 1 and 3 demonstrate relatively unremarkable tracer uptake in both anterior and posterior images, with patients 2 and 4 indicating possible but inconclusive focal tracer uptake.

Both OSEM SPECT and XSPECT Bone reconstructions demonstrate widespread osteoblastic metastases, where xSPECT Bone reconstruction algorithm reveals sharper delineation of vertebral bodies, intervertebral disc spaces and spinous processes (Fig. 2). xSPECT Bone reconstructed images show diffusely intense skeletal uptake with higher visual clarity when compared with the standard OSEM SPECT reconstructed image. Focal tracer uptake shows improved delineation and could be appreciated with higher contrast and resolution on xSPECT/CT fused images (Figs. 3 and 4). Fig. 3 confirmed generalised sclerosis with few focal uptake areas seen in the pelvis, correlated with high resolution on xSPECT/CT images, providing accurate diagnosis. Accurate delineation of sclerotic vertebral lesions (Fig. 4) are shown with high contrast and resolution in XSPECT/CT fused images.

Tracer uptake was confirmed on all hybrid scans with correlation of diffuse bone lesions on CT images and increased tracer uptake on reconstructed OSEM SPECT and XSPECT Bone images. xSPECT/CT images sharply define extensive osteoblastic metastases along with improved clarity of all long bones, skull, ribs and pelvis, demonstrating significant diagnostic value when compared with planar images and OSEM SPECT/ CT (Figs. 2 and 4). In addition, xSPECT/CT reconstructed images were far more superior than OSEM SPECT/CT in showing focal uptake of small osteoblastic lesions (Fig. 3). In patient 1, xSPECT/CT showed focal uptake in the small sclerotic lesion at sacrum, which was negative on SPECT/CT (Fig. 3). Hence, OSEM SPECT/CT may potentially lead to false negative results. Furthermore, skeletal metastases, active degenerative changes and physiological skeletal tracer uptake is well defined and differentiated in XSPECT/CT.
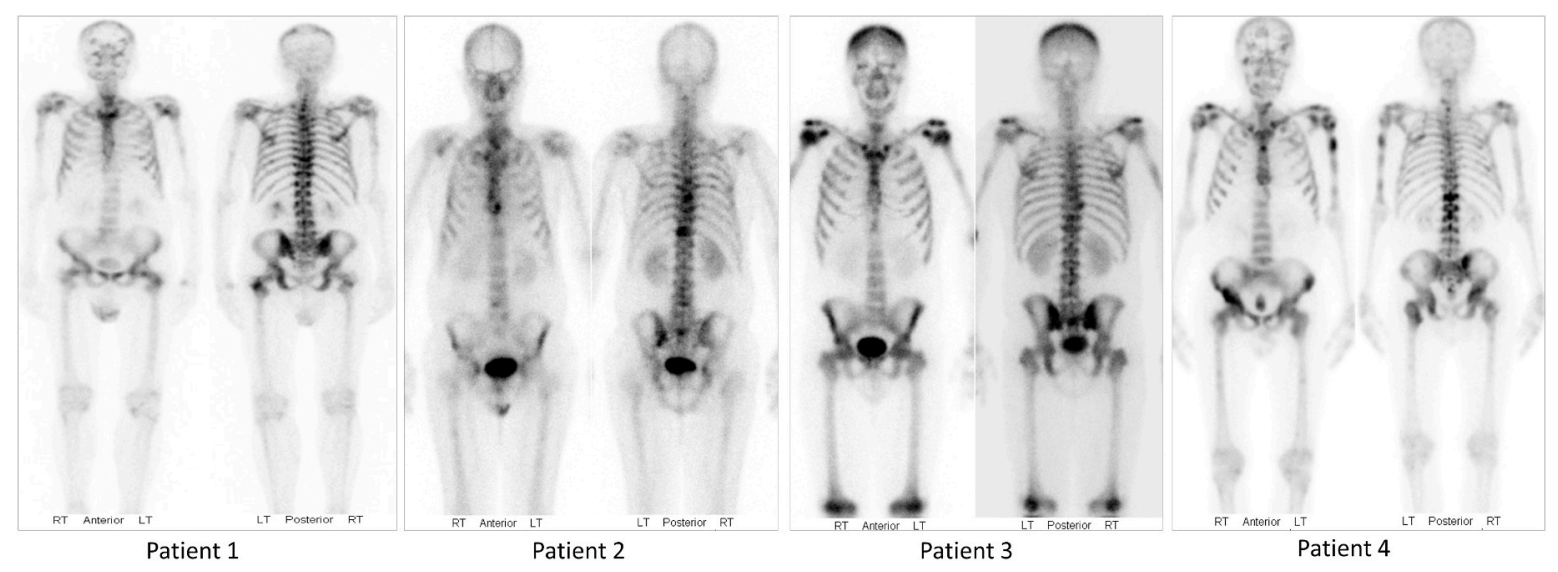

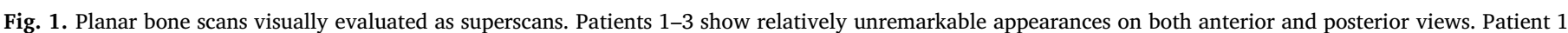

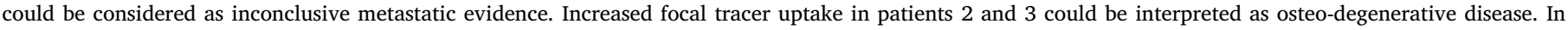
patient 4 , tracer uptake in the humerus could be metastatic evidence, whereas lumbar and pelvic regions demonstrate inconclusive focal tracer uptake. 


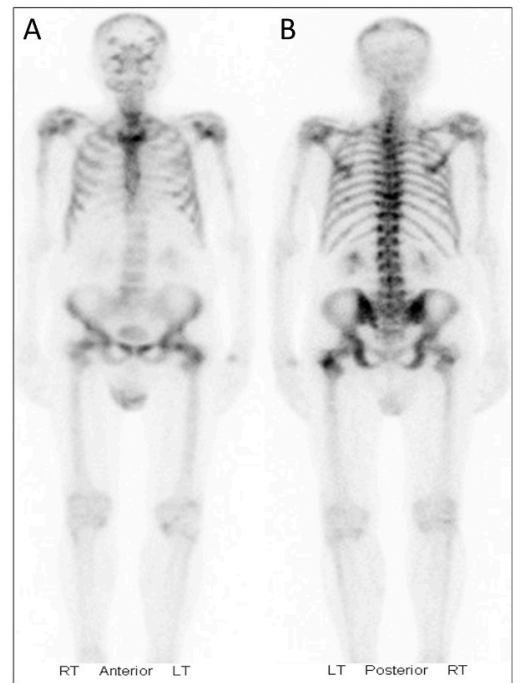

Planar bone scan

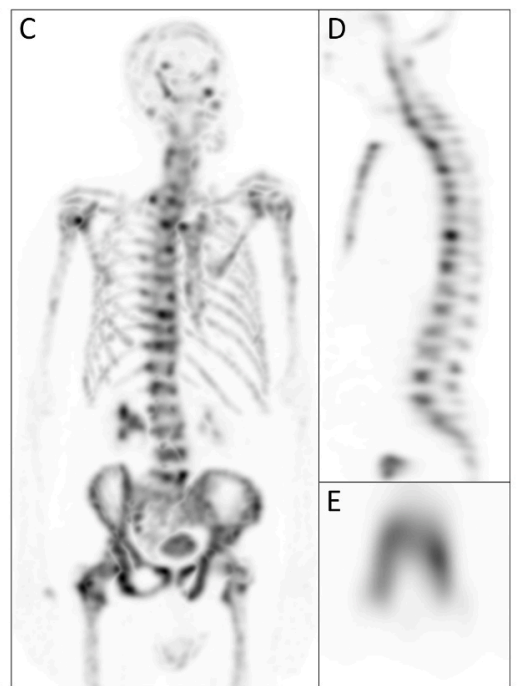

OSEM SPECT

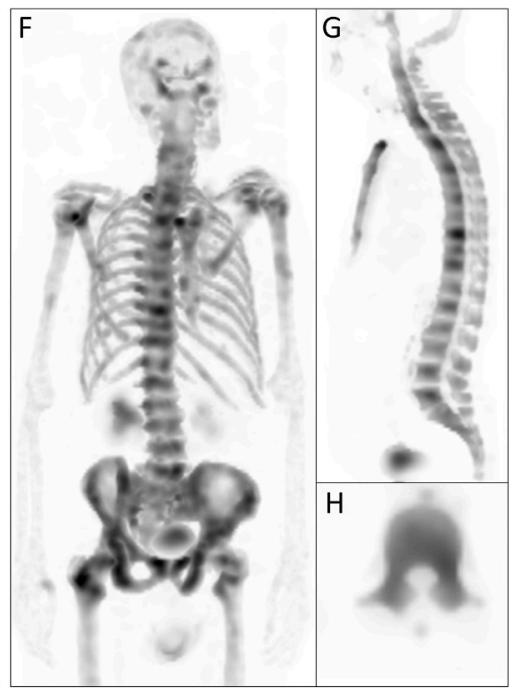

xSPECT Bone

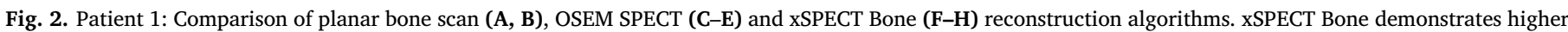
contrast and resolution within metastatic lesions, as well as clear delineation of ribs and pelvis.

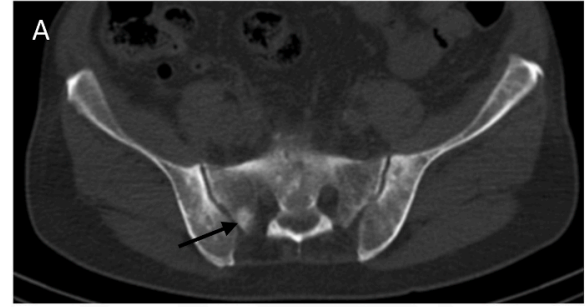

B
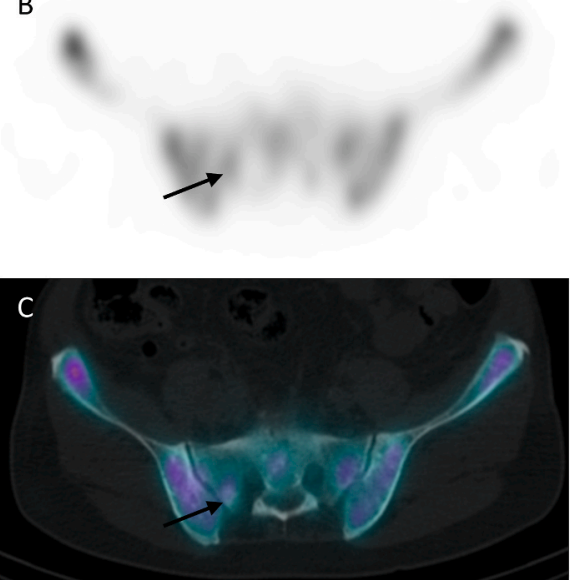

SPECT/CT

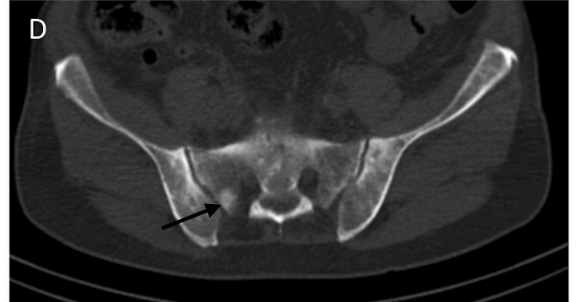

$E$
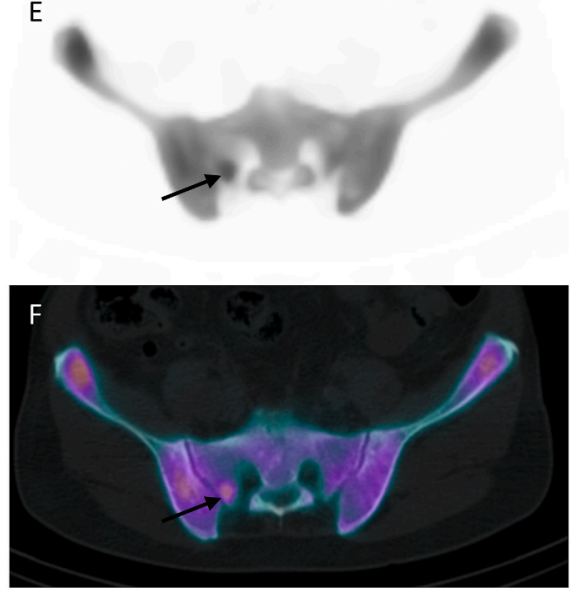

XSPECT/CT

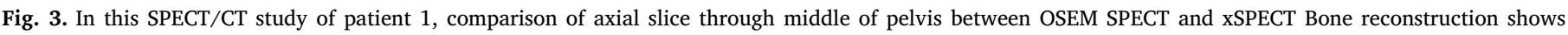

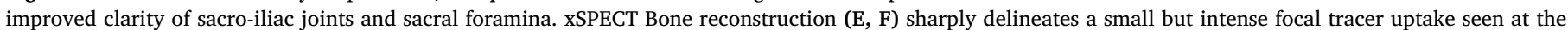
right sacral ala (arrow), compared to OSEM SPECT (B, C); corresponding with sclerotic lesion seen on CT (A, D).

\section{Discussion \& conclusion}

Prostate cancer is the most common condition frequently associated with a superscan (Soloway et al., 1988). A study of 307 prostate cancer patients reported that $15 \%$ demonstrated superscan appearances, of which $72 \%$ of the same group (15\%) had serum PSA levels of $178 \mathrm{ng} / \mathrm{ml}$ and $122 \mathrm{ng} / \mathrm{ml}$ in symptomatic and asymptomatic patients respectively (Manohar et al., 2017). However, the limitation of superscans is that it is unable to distinguish between metabolic bone disorders from skeletal malignancy (Agrawal et al., 2015; Gnanasegaran et al., 2009). Our study also demonstrated that superscans also provide inconclusive skeletal metastases and the inability to delineate the true extent of metastatic disease or small lesions (Fig. 1), thereby affecting patient management.

The xSPECT Bone reconstructed images demonstrated higher contrast and increased resolution between bone and background tracer uptake, resulting in superior diagnostic accuracy. The extent of metastatic evidence is clearly delineated with increased clarity by XSPECT Bone. Skeletal metastases in patient 2 may be demonstrated in planar images (Fig. 1), but the true extent of metastatic disease and intensity of tracer uptake is delineated with higher visual clarity and evaluation on xSPECT Bone reconstructions, further confirmed by corresponding 

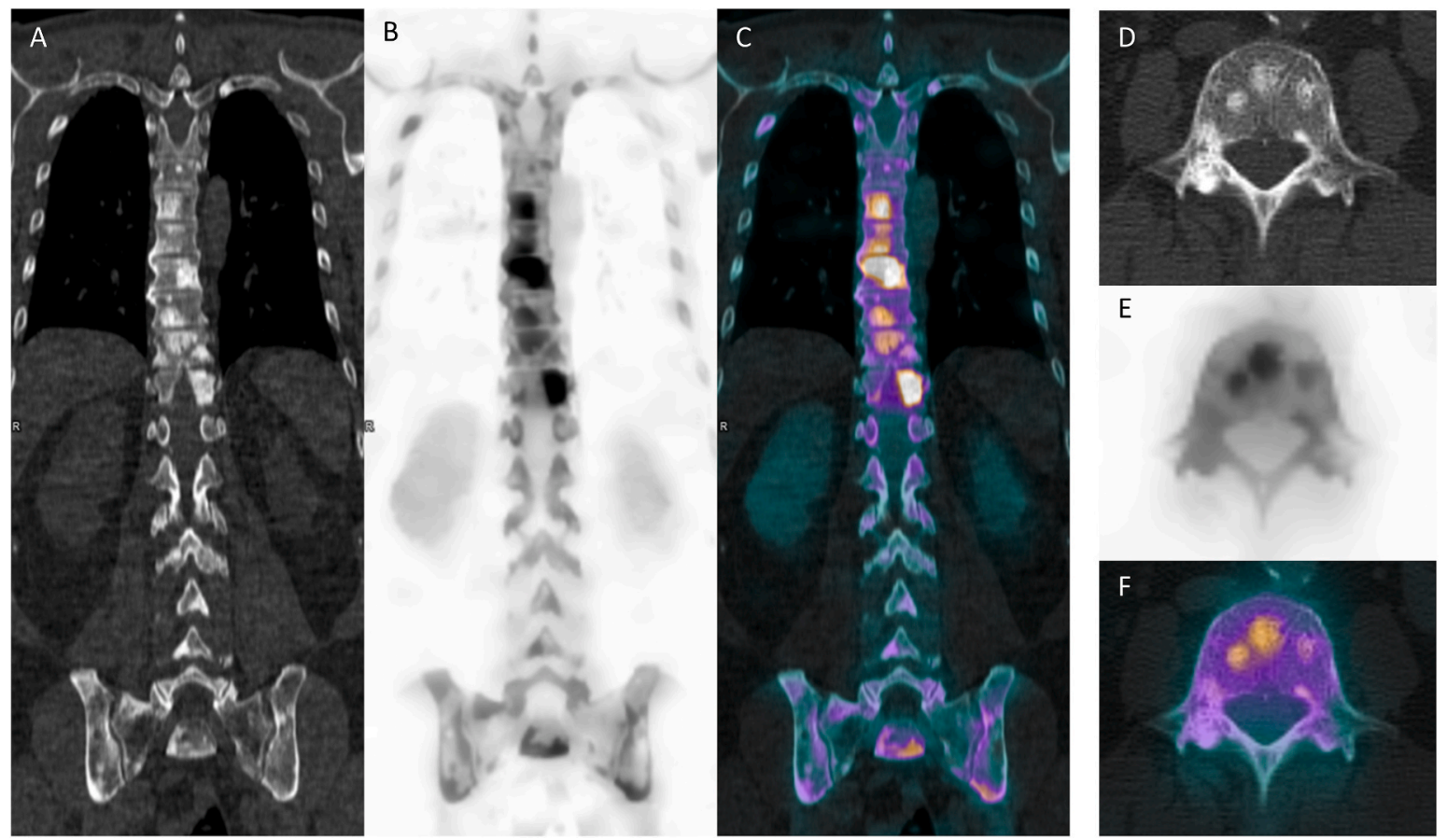

Fig. 4. xSPECT/CT images of patient 2: CT (A, D); xSPECT Bone reconstructed images (B, E); xSPECT/CT fused images (C, F). Sharp delineation and focal tracer uptake clearly shown along specific areas of the spine in xSPECT/CT fused images of patient 2. Focal metastatic lesions are visualised with superior clarity in individual vertebral bodies.

sclerotic lesions seen on CT (Fig. 4).

When compared with standard OSEM SPECT images, xSPECT Bone reconstructed images were far superior in delineating focal areas of osteoblastic metastases (Fig. 2) and improved visualisation of the entire skeletal system, prior to fusing with CT images. The xSPECT Bone images also provided far more diagnostic information when compared with planar images. The superiority of image contrast and resolution in xSPECT Bone provides the ability to resolve any discrepancies that may arise when defining disease progression from metastatic evaluation or non-oncological bone diseases, such as bone remodelling from trauma, physiological tracer uptake or osteo-degenerative changes, even with small lesions (Delcroix et al., 2018; Duncan and Ingold, 2018). This advancement in bone scans could offer clinicians with comprehensive understanding of the true skeletal metastatic burden involved in prostate cancer, especially in detecting tiny skeletal pathologies before progressing to extensive metastasis, as shown in Fig. 3.

Here, we propose that a whole-body (vertex-of-skull to pelvis) xSPECT/CT image reconstruction should be performed in patients with elevated serum PSA levels, along with standard planar imaging. In places that do not have such services, a standard OSEM SPECT image reconstruction should suffice. If patients are in critical pain and unable to endure an extended procedure, a minimum 3D SPECT/CT study should be performed at the region of interest instead of whole-body imaging. If hybrid imaging is not available, a referral to the nearest nuclear medicine department with SPECT/CT modality is advised. This should be performed in order to enhance the diagnostic accuracy of bone scan in staging prostate cancer as disease management, patient outcome and quality of life relies heavily on accurate diagnosis.

To prevent inaccurate staging of other metastatic diseases, this should not only be limited to prostate cancer, as skeletal metastases are evident in other cancers such as breast and lung cancers (Love et al., 2003; Shen et al., 2014; Tabotta et al., 2019). Therefore, by including patient history and key clinical presentation and complaints, the future use of bone scan could drastically improve diagnosis, patient outcome and quality of life by the implementation of hybrid xSPECT/CT imaging.

\section{Declaration of competing interest}

The authors declare that they have no known competing financial interests or personal relationships that could have appeared to influence the work reported in this paper.

\section{Acknowledgment}

We would like to thank all staffs in Nuclear Medicine Department for continuous support and assistance. Also, Sunway Medical Centre for sponsoring of participation in the 2nd International Forum 2019 on Advances in Radiation Physics.

\section{Appendix A. Supplementary data}

Supplementary data to this article can be found online at https:// doi.org/10.1016/j.radphyschem.2020.108998.

\section{References}

Agrawal, K., Marafi, F., Gnanasegaran, G., Van der Wall, H., Fogelman, I., 2015. Pitfalls and limitations of radionuclide planar and hybrid bone imaging. Semin. Nucl. Med. $45,347-372$

Al-Tamimi, A., Tan, A.E., Kwong, S.Y., Sam, C.C., Chong, A., Tan, C.H., 2012. Falsenegative bone scan and choline pet/ct study in a case of prostate cancer: the pitfall of the small cell prostate carcinoma variant. World J. Nucl. Med. 11, 75-78.

Brenner, A.I., Koshy, J., Morey, J., Lin, C., DiPoce, J., 2012. The bone scan. Semin. Nucl. Med. 42, 11-26.

De Visschere, P., Oosterlinck, W., De Meerleer, G., Villeirs, G., 2010. Clinical and imaging tools in the early diagnosis of prostate cancer, a review. JBR-BTR. 93, 62-70.

Delcroix, O., Robin, P., Gouillou, M., Le Duc-Pennec, A., Alavi, Z., Le Roux, P.Y., Abgral, R., Salaun, P.Y., Bourhis, D., Querellou, S., 2018. A new SPECT/CT reconstruction algorithm: reliability and accuracy in clinical routine for non-oncologic bone diseases. EJNMMI Res. 8, 14.

Duncan, I., Ingold, N., 2018. The clinical value of xSPECT/CT Bone versus SPECT/CT. A prospective comparison of 200 scans. Eur. J. Hybrid. Imag. 2, 4.

Gnanasegaran, G., Cook, G., Adamson, K., Fogelman, I., 2009. Patterns, variants, artifacts, and pitfalls in conventional radionuclide bone imaging and SPECT/CT. Semin. Nucl. Med. 39, 380-395.

Halabi, S., Small, E.J., Kantoff, P.W., Kattan, M.W., Kaplan, E.B., Dawson, N.A., Levine, E.G., Blumenstein, B.A., Vogelzang, N.J., 2003. Prognostic model for predicting 
survival in men with hormone-refractory metastatic prostate cancer. J. Clin. Oncol. 21, 1232-1237.

Imbriaco, M., Larson, S.M., Yeung, H.W., Mawlawi, O.R., Erdi, Y., Venkatraman, E.S., Scher, H.I., 1998. A new parameter for measuring metastatic bone involvement by prostate cancer: the Bone Scan Index. Clin. Canc. Res. 4, 1765-1772.

Keller, E.T., Brown, J., 2004. Prostate cancer bone metastases promote both osteolytic and osteoblastic activity. J. Cell. Biochem. 91, 718-729.

Knudson, G., Grinis, G., Lopez-Majano, V., Sansi, P., Targonski, P., Rubenstein, M., Sharifi, R., Guinan, P., 1991. Bone scan as a stratification variable in advanced prostate cancer. Cancer 68, 316-320.

Love, C., Din, A.S., Tomas, M.B., Kalapparambath, T.P., Palestro, C.J., 2003. Radionuclide bone imaging: an illustrative review. Radiographics 23, 341-358.

Manohar, P.R., Rather, T.A., Khan, S.H., Malik, D., 2017. Skeletal metastases presenting as superscan on Technetium $99 \mathrm{~m}$ methylene diphosphonate whole body bone scintigraphy in different type of cancers: a 5-year retro-prospective study. World J. Nucl. Med. 16, 39-44.

Pollen, J.J., Witztum, K.F., Ashburn, W.L., 1984. The flare phenomenon on radionuclide bone scan in metastatic prostate cancer. AJR Am. J. Roentgenol. 142, 773-776. Sabbatini, P., Larson, S.M., Kremer, A., Zhang, Z.F., Sun, M., Yeung, H., Imbriaco, M., Horak, I., Conolly, M., Ding, C., Ouyang, P., Kelly, W.K., Scher, H.I., 1999. Prognostic significance of extent of disease in bone in patients with androgen-independent prostate cancer. J. Clin. Oncol. 17, 948-957.

Shen, G., Deng, H., Hu, S., Jia, Z., 2014. Comparison of choline-PET/CT, MRI, SPECT, and bone scintigraphy in the diagnosis of bone metastases in patients with prostate cancer: a meta-analysis. Skeletal Radiol. 43, 1503-1513.

Siegel, R.L., Miller, K.D., Jemal, A., 2019. Cancer statistics, 2019. Ca - Cancer J. Clin. 69, 7-34.

Soloway, M.S., Hardeman, S.W., Hickey, D., Raymond, J., Todd, B., Soloway, S., Moinuddin, M., 1988. Stratification of patients with metastatic prostate cancer based on extent of disease on initial bone scan. Cancer 61, 195-202.

Tabotta, F., Jreige, M., Schaefer, N., Becce, F., Prior, J.O., Nicod Lalonde, M., 2019. Quantitative bone SPECT/CT: high specificity for identification of prostate cancer bone metastases. BMC Muscoskel. Disord. 20, 619. 